\title{
Deterioro y estrategias de conservación de elementos metálicos de interés patrimonial de la estación de ferrocarril de Aranjuez ${ }^{(\cdot)}$
}

\author{
M. García-Heras*, F. Agua*, V. López**, J. Contreras*** y M.A. Villegas*
}

\begin{abstract}
Resumen
Se ha evaluado el estado de conservación de las marquesinas metálicas de los andenes y terminal de viajeros de la estación de ferrocarril de Aranjuez. Los objetivos incluyen la diagnosis de los procesos y mecanismos de deterioro, degradación y corrosión que han tenido lugar, y el establecimiento de criterios de intervención, rehabilitación y conservación preventiva, adecuados para su correcta preservación. Se determinaron las patologías macroscópicas y microscópicas de los materiales metálicos que, por su naturaleza y protagonismo en las marquesinas, poseen un considerable interés histórico, mediante observaciones de microscopía óptica y microscopía electrónica de barrido de emisión de campo. También se ha llevado a cabo un estudio analítico utilizando difractometría de rayos X, análisis metalográfico y microanálisis por dispersión de energías de rayos X. La evaluación de los resultados obtenidos permitió esclarecer los procesos químico-físicos de degradación, así como el establecimiento de recomendaciones de intervención y pautas de conservación preventiva.
\end{abstract}

\section{Deterioration and conservation strategies of heritage metallic elements of the railway station of Aranjuez}

\begin{abstract}
The conservation state of metallic structures (shelters) of platforms and travellers building of the railway station of Aranjuez has been evaluated. The objectives include the diagnosis of processes and mechanisms of deterioration, degradation and corrosion occurred, as well as the best intervention and preventive conservation criteria for correct preservation. Metallic materials are the real protagonists of the Aranjuez station architectural style and possess notable historical interest. Their macroscopic and microscopic pathologies were determined by using optical microscopy and field emission scanning electron microscopy. Likewise, an analytical study by X-ray diffractometry, metallography and X-ray energy dispersive spectrometry was carried out. Experimental results clarified the chemical-physical degradation processes, the intervention recommendations and preventive conservation guidelines.
\end{abstract}

Keywords

Metallic elements; Corrosion products; Degradation; Conservation; Aranjuez.

\section{INTRODUCCIÓN}

La historia arquitectónica de la estación de Aranjuez está ligada a la de la línea de ferrocarril MadridAranjuez, que se inició en 1845 durante el reinado de Isabel II ${ }^{[1]}$.

José de Salamanca consiguió en 1845 la concesión definitiva para construir la línea MadridAranjuez. La línea se inauguró el 9 de febrero de 1851 en la Estación Embarcadero de Atocha. Los materiales metálicos de esta estación fueron suministrados por Fundición de Hierro y Construcciones de Máquinas Sanford, sita en el Paseo de Recoletos de Madrid ${ }^{[2]}$ y La Constancia, empresa marbellí de Heredia, cuñado del Marqués de Salamanca. En el proyecto de nueva estación de 1908 el coste de las marquesinas ascendía a $6.800 \mathrm{Pta}^{[3]}$ y el material base hierro era el protagonista, incluso por su presencia estructural en pilares y vigas a la vista, así como en elementos ornamentales ${ }^{[4]}$.

En 1914 se proyecta una nueva estación cuyas obras, presupuestadas a fecha de 1915 en 600.000 Pta,

\footnotetext{
(•) Trabajo recibido el día 27 de Enero de 2010 y aceptado en su forma final el día 5 de Mayo de 2010.

* Instituto de Historia. Centro de Ciencias Humanas y Sociales, CSIC.Calle Albasanz, 26-28. 28037 Madrid. Autor para envío de correspondencia: mariangeles.villegas@cchs.csic.es.

** Centro Nacional de Investigaciones Metalúrgicas, CENIM, CSIC. Avenida Gregorio del Amo, 8. 28040 Madrid.

*** Arquitecto. Calle Valderribas, 12. 28007 Madrid.
} 
comprendían una importante partida sobre marquesinas. Este proyecto se ejecutó en 1922. Los planos del proyecto se aprobaron en 1923, siendo Narciso Clavería el arquitecto. Clavería ya había realizado en 1917 la nueva estación de Toledo de estilo Neomudéjar ${ }^{[5]}$, que supuso el referente directo para la estación de Aranjuez. La estación de Aranjuez guarda una fusión entre el estilo Neomudéjar propio de las arquitecturas ferroviarias (verdaderos prototipos de la Compañía Madrid-Zaragoza-Alicante, MZA) y las nuevas tendencias industriales de porte metálico. En la figura 1 se puede observar una fotografía de 1927 de la nueva estación de Aranjuez recién terminada, con la gran marquesina que se extiende sobre el andén y la fachada sur del edificio principal. Actualmente, junto al nuevo edificio de viajeros, hay tres andenes protegidos por otras tantas marquesinas y unidos por un paso inferior de $32 \mathrm{~m}$ de longitud.

En el presupuesto final del proyecto de 1922 de la Sociedad de Fomento de Obras y Construcciones, se especificaba que la armadura para la cubierta iría formada por correas de hierro laminado en doble T, apoyadas sobre los piñones de la fábrica de ladrillo. En la instalación de las marquesinas participaron las empresas: Taller Central de Vía y Obras de Villaverde Bajo (aleaciones base hierro), Casa de Juan Donato y Franco (vidrios) y Uralita S.A. (Uralita). Aunque el proyecto y la memoria original sobre las marquesinas no están documentados, se ha podido seguir el proceso de su construcción ${ }^{[4]}$. En 1924 el Taller Central de Villaverde había aumentado su producción hasta $3.400 \mathrm{Tm}$, de las que $600 \mathrm{Tm}$ correspondían a las marquesinas de la estación de Aranjuez. Las marquesinas que se elaboraron fueron de un tipo que

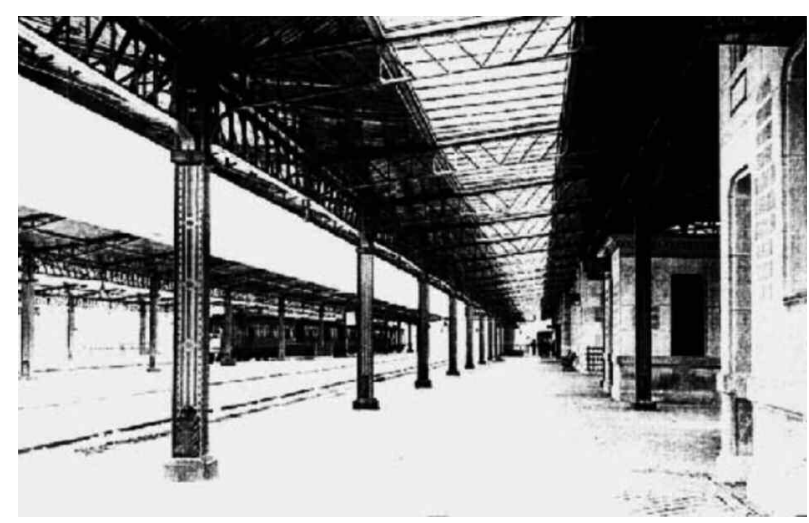

Figura 1. Imagen de 1927 de la llamada nueva estación de Aranjuez, realizada según el proyecto de 1923.

Figure 1. Image of 1927 of the so-called new station of Aranjuez, built after the project of 1923. posteriormente se aplicó para otras estaciones. El precio superó las 300.000 Pta.

En la figura 2 se muestra el detalle de la decoración de los pilares de las marquesinas de la estación de Algodor, realizadas sobre los diseños originales de los pilares de la estación de Aranjuez. Puede comprobarse su estrecha similitud con las fotografías actuales de las marquesinas de Aranjuez.

En junio de 1939, tras la Guerra Civil, se acometieron las reparaciones pertinentes y en la memoria de 1941 de los edificios reparados después de la contienda figuran las marquesinas y el lucernario, que se habían reparado en 1940.

El presente trabajo tiene por objeto la caracterización del estado de conservación de los materiales metálicos de las marquesinas de la estación de ferrocarril de Aranjuez.

Los objetivos concretos son: la evaluación arqueométrica de los materiales objeto de estudio; la diagnosis de los procesos y mecanismos químico-físicos de deterioro, degradación y corrosión que han tenido lugar en dichos materiales; y el establecimiento de criterios de intervención, restauración y conservación preventiva adecuados para una correcta preservación de las marquesinas.

\section{PROCEDIMIENTO DE TRABAJO}

La primera aproximación a los elementos metálicos objeto de estudio, se realizó mediante una inspección visual, en la que se apreció su estado de conservación general a nivel macroscópico. Durante dicha inspección se tomaron las muestras representativas

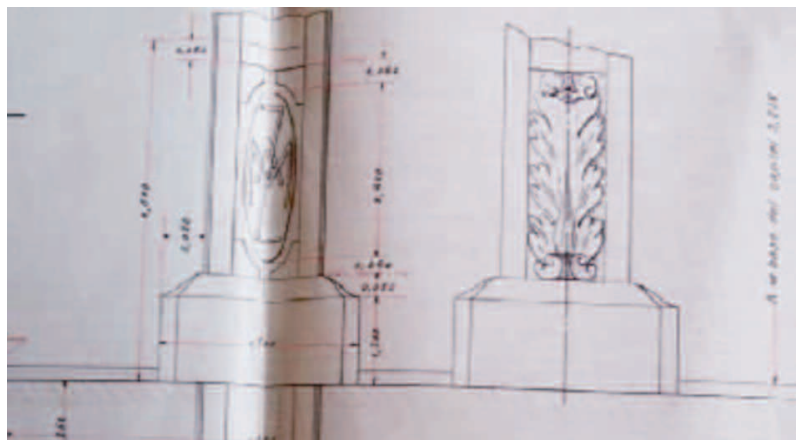

Figura 2. Esquema de los motivos decorativos de la base de los pilares de las marquesinas de la estación de Algodor, basados en los correspondientes de la estación de Aranjuez.

Figure 2. Schema of ornamental bottom designs in the cantilever roof pillars of the Algodor's station, based on those of the Aranjuez station. 
necesarias para llevar a cabo la caracterización de la degradación de dichos materiales, así como los análisis que permitieron esclarecer los mecanismos químico-físicos de corrosión y sugerir los criterios de intervención y conservación.

Se seleccionaron muestras de acero procedentes de un pilar (moldura desprendida del pilar 14 del andén B), de una pieza de fundición de la parte superior de un pilar, que se hallaba completamente desprendida y caída (pilar 2 del andén B, cara exterior), y diversas piezas de costra de corrosión tomadas cerca de la base del pilar 1 del andén A. Las figuras 3 A), B), D) y E), muestran el aspecto de los fragmentos seleccionados.

El espesor medio estimado de las costras de corrosión desprendidas es $\approx 3$ y $4 \mathrm{~mm}$.

En la moldura de acero dicho espesor es de apenas $1 \mathrm{~mm}$.

Para efectuar el examen microscópico se utilizó un microscopio estereoscópico convencional de luz reflejada Olympus modelo DP-11.
Las observaciones de microscopía electrónica de barrido de emisión de campo (MEBEC), se llevaron a cabo con un microscopio Jeol modelo JSM 6500F a $15 \mathrm{kV}$.

Las muestras se estudiaron en el estado de recepción sin manipulación posterior y sobre probeta pulida metalográficamente. Previamente fueron recubiertas con carbono como medio conductor. Los microanálisis por espectroscopía de dispersión de energías de rayos $\mathrm{X}$ (EDX), se efectuaron con un espectrómetro Pentafet Link acoplado al microscopio.

Para realizar el análisis metalográfico se cortó un fragmento de las muestras de acero y de fundición de hierro, que posteriormente fue embutido en baquelita y se preparó para metalografía mediante desbaste con papeles abrasivos de carburo de silicio y pulido posterior en dos etapas con pasta de diamante de $3 \mathrm{y}$ $1 \mu \mathrm{m}$, respectivamente. El examen microscópico se efectuó sobre probeta pulida sin ataque metalográfico y en probetas pulidas y atacadas con el reactivo
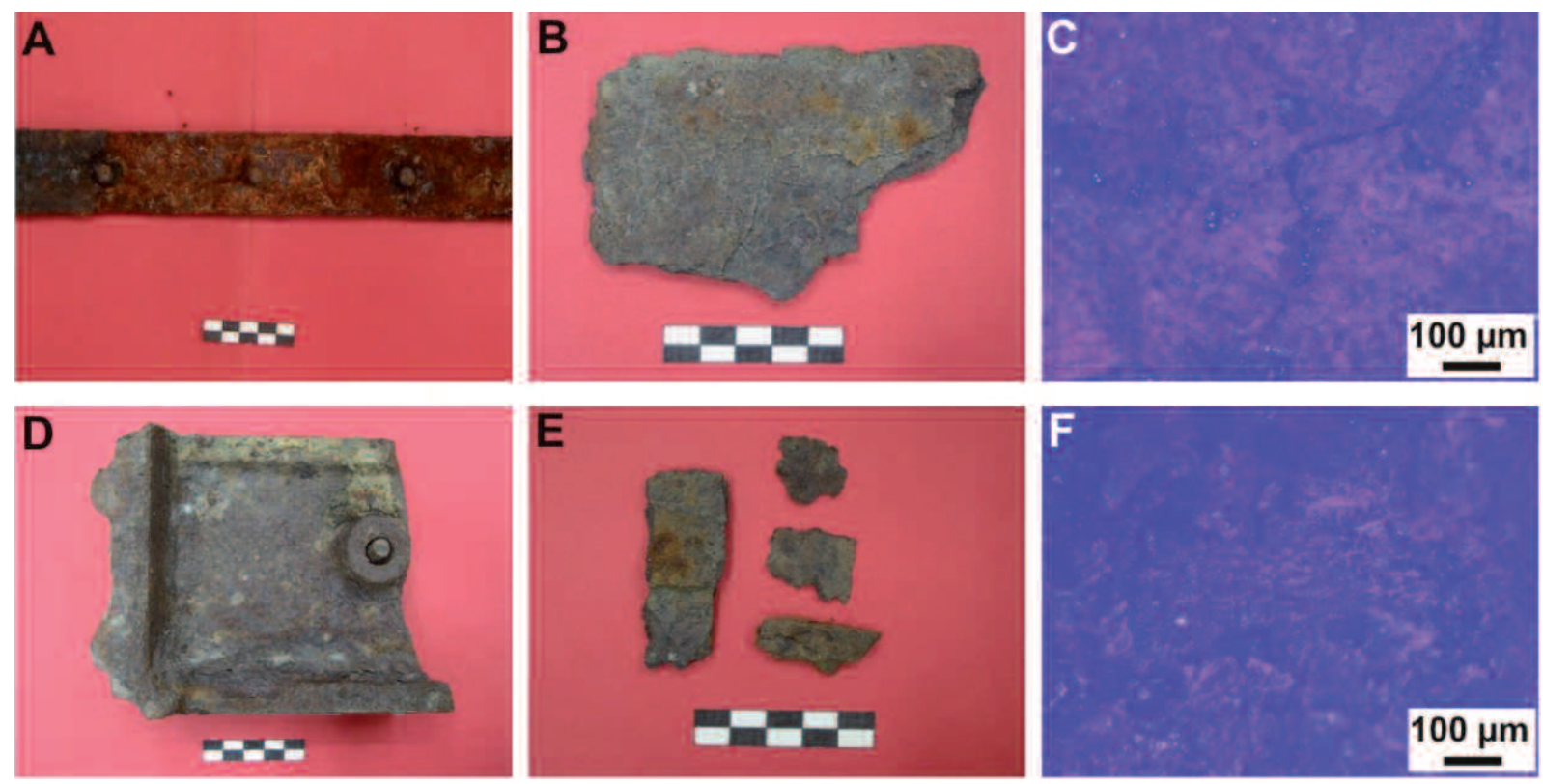

Figura 3. A) Moldura de acero desprendida del pilar 14 del andén B en el extremo Alicante, cara interna. B) Fragmento de costra de corrosión (muestra costra 1) tomado en el pilar 1 del andén A en extremo Madrid. C) Imagen de MO de la cara externa de una muestra de costra de corrosión. D) Fragmento de pieza de fundición superior del pilar 2 del andén $B$, cara interna. E) Fragmentos de costras de corrosión (muestras costras 2) tomados en el pilar 1 del andén A en extremo Madrid, cara interna. F) Imagen de MO de la cara interna de una muestra de costra de corrosión.

Figure 3. A) Steel moulding detached from pillar 14 of platform $B$ at the Alicante end, internal side. B) Corrosion crust fragment (sample crust 1) taken from pillar 1 of platform A at the Madrid end. C) OM image of the external side of a corrosion crust sample. D) Fragment of one cast iron upper piece of pillar 2 of platform B, internal side. E) Corrosion crust fragments (sample crust 2) taken from pillar 1 of platform $A$ at the Madrid end, internal side. F) OM image of the internal side of a corrosion crust sample. 
metalográfico Nital $2 \%$ (disolución de 98 \% en volumen de etanol $\mathrm{CH}_{3}-\mathrm{CH}_{2} \mathrm{OH}$ y $2 \%$ en volumen de ácido nítrico $\mathrm{HNO}_{3}$ concentrado). Las observaciones metalográficas y la toma de micrografías se efectuaron con un microscopio metalográfico Olympus modelo PME3.

Los difractogramas de rayos X (DRX) de las muestras de costra de corrosión se registraron con un equipo Siemens modelo D-5000, radiación Ka del cobre $(\mathrm{Cu}, 1,54060 \AA)$, bajo condiciones de trabajo de $40 \mathrm{kV}$ y $30 \mathrm{~mA}$. Los difractogramas se obtuvieron con una velocidad angular de $0,4^{\circ} / \mathrm{min}$ entre $2 \theta=10-80^{\circ}$.

\section{RESULTADOS}

\subsection{Inspección visual. Estado de conservación general}

El conjunto de las marquesinas presenta un aspecto muy deteriorado que se hace especialmente grave en los elementos metálicos de los pilares, vigas y correas. La figura 4 A) muestra una perspectiva de las marquesinas de los andenes secundarios desde el andén principal del edificio de viajeros. La figura 4 B) representa el esquema de alzado de un pilar, donde se han indicado las diversas partes a las que hace referencia el presente trabajo.
El andén A está situado en el extremo sur de la estación, en la parte más alejada del edificio de viajeros. El alero de la cubierta en su extremo Madrid presenta faltas de elementos decorativos y signos de corrosión. En la figura 5 A) se aprecia la acusada inclinación del pilar 1 y en la figura 5 B) la intensa corrosión de todos los elementos del pilar 3. Una patología especial son las perforaciones de magnitudes muy considerables, como la que se puede observar en la figura 5 C) de la parte inferior del pilar 6 .

En general, los aleros de la cubierta del andén B se encuentran en mejor estado de conservación que los del andén A, aunque presentan las mismas patologías. En la figura 5 D), correspondiente a la parte superior del pilar 14, se observan los productos de corrosión por debajo de la capa desprendida de pintura. En esa imagen se ha indicado con una flecha, la moldura desprendida que se extrajo para la toma de muestras de acero.

Los elementos de las marquesinas del andén C (andén del edificio de viajeros) muestran un estado de conservación menos degradado que los de los otros andenes.

Los aleros de la cubierta mantienen los elementos decorativos, aunque por su cara interior presentan signos de corrosión avanzada. La figura $5 \mathrm{E}$ ) muestra el hundimiento y apuntalamiento del pilar 6 .

Los andenes D y E se encuentran a la altura de la parte delantera del edificio de viajeros. Su estado de conservación general es variable. En la esquina de
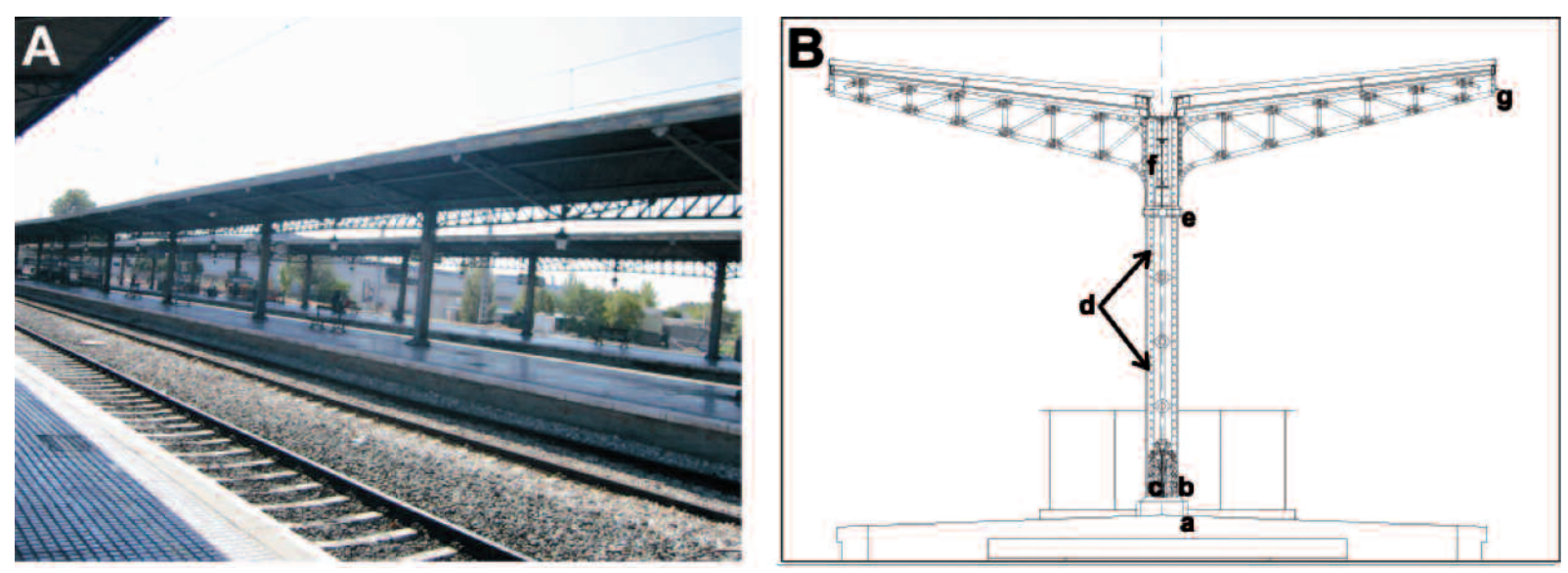

Figura 4. A) Marquesinas de los andenes secundarios desde el andén principal (octubre, 2007. Fotografía M. García-Heras). B) Alzado de un pilar y diversas partes de referencia: a) Base; b) Zona inferior; c) Fundiciones inferiores; d) Molduras de acero; e) Fundiciones superiores; f) Zona superior; g) Vigas y correas adyacentes al pilar.

Figure 4. A) Image of secondary platform cantilever roofs from the main platform (October, 2007. Photograph by M. García-Heras). B) Draw of a pillar and its several parts: a) Base; b) Bottom part; c) Cast iron pieces (bottom); d) Steel mouldings; e) Cast iron pieces (upper); f) Upper part; g) Girders and other metallic elements adjacent to a pillar. 

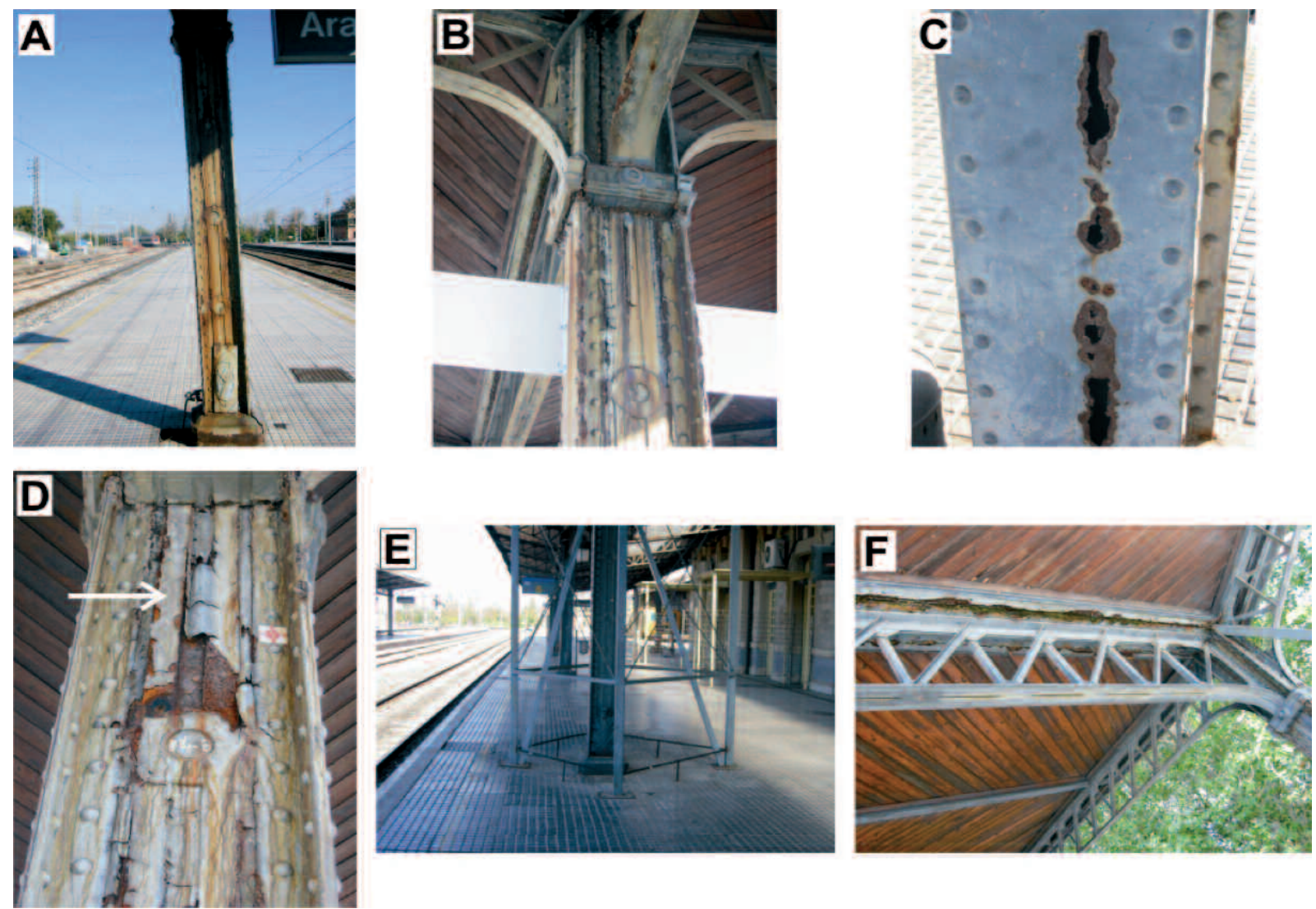

Figura 5. Imágenes de las principales patologías detectadas en las marquesinas de los andenes (octubre, 2007. Fotografías M. García-Heras). A) Pilar 1 del andén A. B). Parte superior del pilar 3 del andén A. C). Parte inferior del pilar 6 del andén A. D) Pilar 14 del andén B, la flecha indica la moldura desprendida que se extrajo para la toma de muestras de acero. E) Apuntalamiento y hundimiento del pilar 6 del andén C. F) Correas adyacentes al pilar 15 del andén $\mathrm{E}$.

Figure 5. Images of the main pathologies detected in the platform cantilever roofs (October, 2007. Photographs by M. Garcia-Heras). A) Pillar 1 of platform A. B) Upper part of pillar 3 of platform A. C) Bottom part of pillar 6 of platform A. D) Pillar 14 of platform B, the arrow indicates the moulding taken for selection of steel samples. E) Propping and sinking of pillar 6 of platform C. F) Metallic elements adjacent to pillar 15 of platform E.

unión entre los andenes $\mathrm{C}$ y D se observa un estado deteriorado y perfiles parcialmente desprendidos. La cubierta entre los andenes D y E en el extremo Alicante, está hundida y parcialmente desprendida. Las correas adyacentes a los pilares están intensamente corroídas e incluso algunas presentan musgo (Fig. 5 F)). El lucernario entre los andenes D y E muestra correas corroídas y parcialmente desprendidas.

\subsection{Detección de patologías macro y microscópicas}

Las patologías concretas de todos los pilares y correas de las marquesinas de todos los andenes se contabilizaron mediante inspección visual. De acuerdo con dichas patologías se ha realizado una estimación que se muestra en la tabla I.

Mediante microscopía estereoscópica convencional de luz reflejada se observó la textura y morfología superficial de las muestras de costra de corrosión. En las figuras $3 \mathrm{C}$ ) y F) se pueden apreciar algunas imágenes de las muestras observadas. Las caras externas tienen un aspecto rugoso, heterogéneo, con abundantes zonas rojizas y algunas otras negruzcas. También se observan algunas microgrietas. En las caras internas la mayoría de las superficies se hallan cubiertas de depósitos rojizos con menos zonas negruzcas. El aspecto también es muy heterogéneo, pero no se observaron microgrietas. 
DETERIORO Y ESTRATEGIAS DE CONSERVACIÓN DE ELEMENTOS METÁLICOS DE INTERÉS PATRIMONIAL DE LA ESTACIÓN DE FERROCARRIL DE ARANJUEZ DETERIORATION AND CONSERVATION STRATEGIES OF HERITAGE METALLIC ELEMENTS OF THE RAILWAY STATION OF ARANJUEZ

Tabla I. Porcentajes de patologías concretas y faltas detectadas en diversas partes de los elementos metálicos de las marquesinas

Table I. Percentages of particular pathologies and lacks detected in different parts of metallic elements of the cantilever roof

\begin{tabular}{|c|c|c|c|c|c|c|c|c|c|c|}
\hline \multirow{3}{*}{ Andén } & \multicolumn{7}{|c|}{ Pilares } & \multirow{3}{*}{$\begin{array}{c}\text { Faltas } \\
\text { de } \\
\text { piezzs } \\
\text { de } \\
\text { fundición }\end{array}$} & \multicolumn{2}{|c|}{ Corrosión en correas } \\
\hline & \multicolumn{2}{|c|}{ Con corrosión } & \multirow{2}{*}{$\begin{array}{c}\text { Con } \\
\text { inclinación }\end{array}$} & \multirow{2}{*}{$\begin{array}{c}\text { Con hundimiento } \\
\text { y/o } \\
\text { apuntalamiento }\end{array}$} & \multirow{2}{*}{$\begin{array}{c}\text { Con } \\
\text { abombamiento }\end{array}$} & \multirow{2}{*}{$\begin{array}{c}\text { Con } \\
\text { picaduras }\end{array}$} & \multirow{2}{*}{$\begin{array}{c}\text { Con } \\
\text { perforaciones }\end{array}$} & & \multirow{2}{*}{$\begin{array}{c}\text { Muy } \\
\text { avanzada }\end{array}$} & \multirow{2}{*}{ Avanzada } \\
\hline & Avanzada & Media & & & & & & & & \\
\hline A & 15 & 27 & 7 & 0 & 20 & 7 & 13 & 8 & 15 & 15 \\
\hline B & 63 & 12 & 0 & 7 & 0 & 0 & 13 & 20 & 36 & 21 \\
\hline C & 13 & 7 & 7 & 20 & 0 & 0 & 13 & 18 & 7 & 7 \\
\hline D & 17 & 21 & 0 & 33 & 0 & 0 & 0 & 14 & 20 & 20 \\
\hline $\mathrm{E}$ & 67 & 0 & 0 & 66 & 0 & 0 & 0 & 28 & 0 & 50 \\
\hline
\end{tabular}

\subsection{Análisis metalográfico}

En la figura 6, imágenes A) y B), se recogen las micrografías correspondientes a la muestra de fundición. Se trata de una fundición gris de matriz perlítica de elevada resistencia mecánica con estructura metalográfica muy homogénea. Presenta una costra de corrosión generalizada en forma de capa distribuida por toda la superficie (Fig. 6 A), zona a)), así como fenómenos de corrosión selectiva (corrosión grafítica) que se visualizan como rosetas (Fig. $6 \mathrm{~A}$ ), zona b)), en líneas de avance hacia el interior de la muestra sin atacar, como consecuencia de que el grafito es catódico (más noble) frente a la ferrita. El espesor medio de la costra de corrosión se estima en unos $0,15 \mathrm{~mm}$. Este resultado indica que las piezas de fundición han estado sometidas a una corrosión muy intensa. Las condiciones climáticas de Aranjuez (temperatura, humedad relativa, presión atmosférica, etc.) no podrían explicar la formación de semejantes alteraciones y degradación química, salvo si se hubiera producido la incidencia de ambientes contaminados, como los que suelen ser frecuentes en zonas industriales. La presencia simultánea de elevada humedad relativa y gases contaminantes de propiedades ácidas (por ejemplo, $\mathrm{SO}_{2}, \mathrm{NO}_{\mathrm{x}}, \mathrm{CO}_{2}$, etc.), podría ser la responsable del ataque químico que ha sufrido la superficie de las piezas de fundición, originando una corrosión intensa y generalizada a toda la superficie de la pieza.

En la figura $6 \mathrm{~B}$ ) se puede apreciar la estructura perlítica de la fundición (zonas p) con la típica morfología laminar, la fase ferrítica más clara (zonas f) y la presencia de eutéctico fosforoso segregado interdendríticamente en la matriz metálica de la fundición de hierro (zonas e) y enriquecido en fósforo (P).
El análisis metalográfico de la muestra de acero de los pilares, indicó que responde a la estructura de un acero no aleado de bajo contenido en carbono $(\mathrm{C} \approx 0,1 \%$ en masa). Se trata de un acero común laminado en caliente que ha experimentado un proceso de alteración y corrosión química que avanza en forma de ondas, como es usual en este tipo de materiales metálicos. La figura $6 \mathrm{C}$ ) presenta una micrografía correspondiente a la muestra de acero, donde se aprecia una zona oscura de productos de corrosión (zona a) y otra zona clara del acero (zona b). Dicha zona clara corresponde a la parte metálica no afectada por la corrosión. Por otro lado, en la figura 6 D) se puede observar la superficie del acero previamente atacada con Nital, donde se resalta la microestructura de los cristales de ferrita de tono claro y pequeñas lagunas grises que corresponden a la perlita, que se encuentra en menor proporción.

Respecto a la corrosión de la muestra de acero, según se deduce del análisis metalográfico, los pilares también han estado sometidos a la acción de un ambiente agresivo (tipo industrial y/o contaminado y combinado con humedad relativa elevada), que ha favorecido un avance intenso de la corrosión. La costra de corrosión que aparece en la figura 6 C) es sólo la parte adherida al acero y, probablemente, se trate de una costra formada en ataques reiterativos, ya que se comprobó que las costras más gruesas ya están desprendidas y tienen 3-4 $\mathrm{mm}$ de espesor medio (ver apartado 2 y Fig. 3).

\subsection{Observaciones de MEBEC y microanálisis EDX}

En la muestra de fundición de hierro se observa (Fig. 7) una costra de corrosión ancha (zona 1) de la 

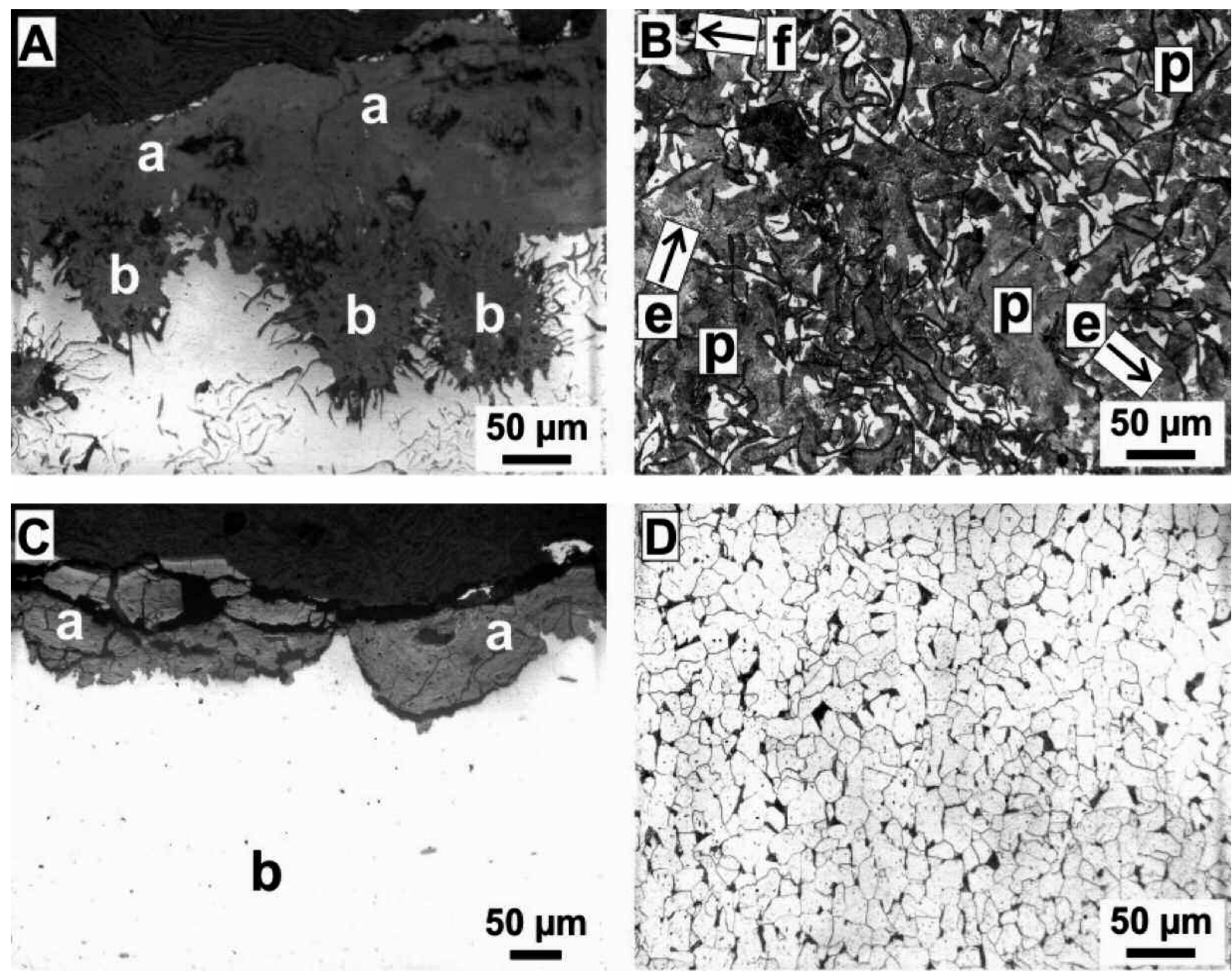

Figura 6. Micrografías metalográficas. A) Muestra de fundición, sección transversal de la superficie con corrosión grafítica. B) Muestra de fundición, morfología de la parte interna (atacada con Nital). C) Muestra de acero, sección transversal de la superficie con avance de corrosión en forma de ondas. D) Muestra de acero, morfología de la parte interna (atacada con Nital).

Figure 6. Metallographic micrographs. A) Cast iron sample, cross section of the surface with graphitic corrosion. B) Cast iron sample, internal side morphology (attacked with Nital). C) Steel sample, cross section of the surface showing waved corrosion advance. D) Steel sample, internal side morphology (attacked with Nital).

que se destacan rosetas de corrosión selectiva (zona 2), que penetran en el cuerpo inalterado de la fundición (zona 3). Los correspondientes análisis químicos por EDX de las tres zonas descritas se indican en la tabla II. Como era de esperar, las zonas 1 y 2 contienen productos de corrosión que pueden proceder del medio ambiente externo (sodio, azufre, potasio y calcio), quedando atrapados dentro de la costra de corrosión y además un elevado porcentaje de oxígeno, asimilable a los óxidos e hidróxidos de hierro formados (diversos compuestos y especies químicas de Fe (II) y/o Fe (III)). La presencia de contenidos altos de silicio en la zona 2 también se debe a un efecto del proceso de corrosión y acumulación de productos procedentes del ambiente exterior. Los porcentajes de hierro en las zonas 1 y 2 son sensiblemente más bajos que en el material inalterado debido a la presencia mayoritaria del oxígeno de los óxidos formados. El análisis químico de la zona 3 responde a la composición que se espera para la fundición gris inalterada.

En la muestra de acero se observa (Fig. 8 A)) una costra de corrosión microfisurada de espesor irregular (zonas 1 y 3 ) y el cuerpo inalterado del acero, donde se aprecian los cristales de ferrita (zona 2). Los resultados de los análisis químicos efectuados por EDX se resumen en la tabla III. En las zonas 1 y 3 de la costra de corrosión se detecta un porcentaje elevado de oxígeno atribuido a los óxidos formados, así como otros productos de la alteración química 


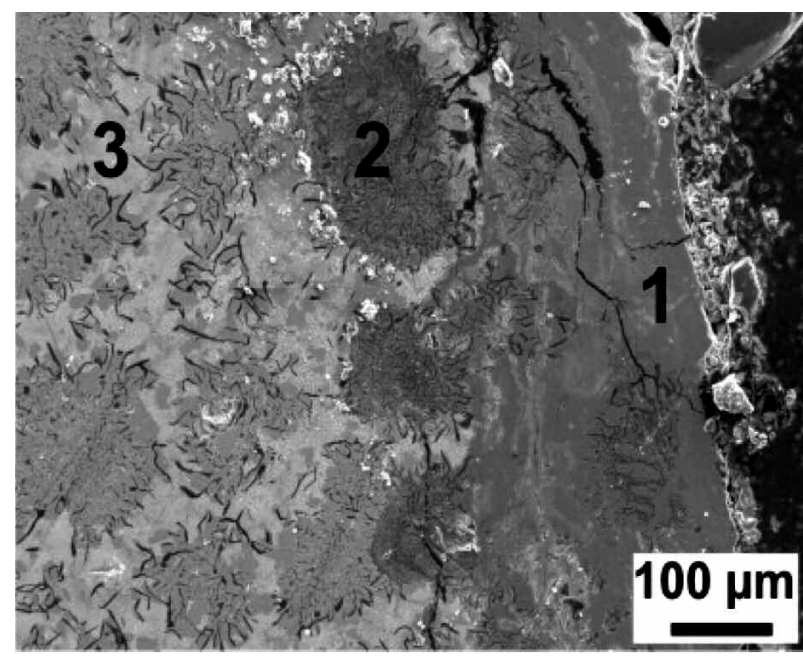

Figura 7. Micrografía de MEBEC de una sección transversal de la muestra de fundición.

Figure 7. FESEM micrograph of the cross section of the cast iron sample.

(azufre y calcio) en proporciones bajas en la zona 3. La escasez de elementos relacionados con las alteraciones y depósitos de la corrosión sólo puede explicarse por la formación de costras sucesivas que se han desprendido al superar el espesor crítico, como ya se ha indicado anteriormente, y/o por el efecto de lavado de las abundantes precipitaciones de lluvia que caracterizan la zona de Aranjuez.

Las observaciones de la superficie de las costras de corrosión por su cara interna (cara más abrigada del aire, pero desprendida de la matriz de acero) indi-
Tabla II. Resultados de microanálisis EDX de la muestra de fundición de la figura 7 (\% en peso atómico)

Table II. Results of EDX microanalyses from the cast iron sample of figure 7 (atomic wt. \%)

\begin{tabular}{cccccccccc} 
Zona & $\mathbf{O}$ & $\mathbf{N a}$ & $\mathbf{S i}$ & $\mathbf{P}$ & $\mathbf{S}$ & $\mathbf{K}$ & $\mathbf{C a}$ & $\mathbf{M n}$ & $\mathbf{F e}$ \\
\hline 1 & 64,8 & 0,5 & 0,7 & - & 0,4 & - & 0,2 & - & 33,4 \\
2 & 62,5 & 0,8 & 17,3 & - & 0,4 & 0,4 & - & - & 18,6 \\
3 & - & - & 4,7 & 1,0 & - & - & - & 1,0 & 93,3
\end{tabular}

Tabla III. Resultados de microanálisis EDX de la muestra de acero de la figura 8 (\% en peso atómico)

Table III. Results of EDX microanalyses from the steel sample of figure 8 (atomic wt. \%)

\begin{tabular}{ccccccc}
\hline Zona & $\mathbf{O}$ & $\mathbf{S i}$ & $\mathbf{S}$ & $\mathbf{C a}$ & $\mathbf{M n}$ & $\mathbf{F e}$ \\
\hline 1 & 61,0 & - & - & - & - & 39,0 \\
2 & - & $\overline{-}$ & $\overline{-}$ & $\overline{-}$ & 0,8 & 99,2 \\
3 & 61,0 & 0,2 & 0,2 & 0,4 & 0,4 & 37,8
\end{tabular}

can una textura rugosa y heterogénea (Fig. 8 B)). Cuando esta zona se observa a mayores aumentos se aprecian formaciones más angulosas, probablemente debidas a microcristales. En el correspondiente micro-
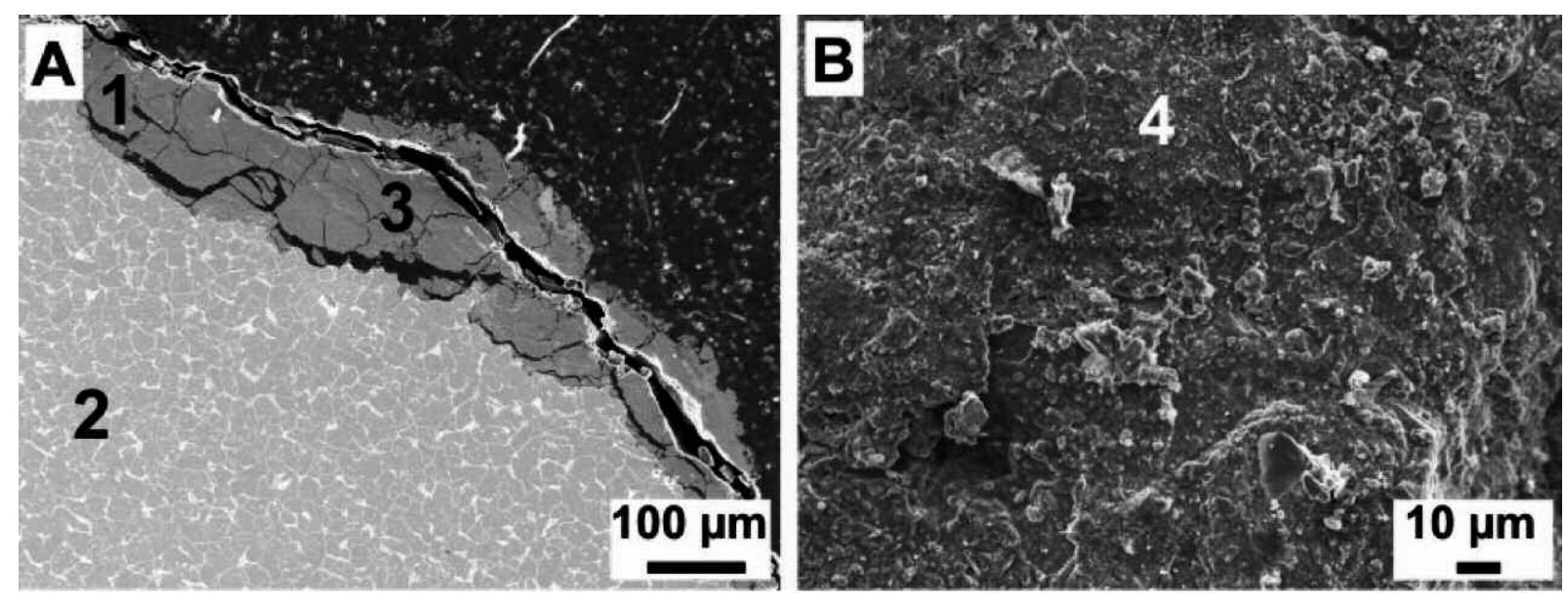

Figura 8. Micrografías de MEBEC. A) Sección transversal de la muestra de acero. B) Superficie de una costra de corrosión (cara interior).

Figure 8. FESEM micrographs. A) Cross section of the steel sample. B) Corrosion crust surface (internal side). 
análisis EDX de esta zona, tabla III, además de un contenido muy elevado de oxígeno asociado a los óxidos formados, se detectan otros productos de la corrosión depositados en la superficie de la costra (azufre y calcio).

Las observaciones de la superficie de las costras de corrosión por su cara externa (cara al aire), indican un aspecto distinto al de la superficie de la cara interna. La superficie aparece también heterogénea, pero menos rugosa y más ondulada, con pequeños fragmentos desprendidos y depositados. También se observan microgrietas de distinta profundidad y anchura. La superficie aparece en buena parte cubierta de formaciones filamentosas irregulares y entramadas. Los resultados de los microanálisis EDX pusieron de manifiesto que ambas zonas presentan óxidos de hierro (diversos óxidos de Fe (II) y/o Fe (III)), si bien en la zona de fondo se detectan además cantidades muy pequeñas de aluminio y azufre. Dichos porcentajes se pueden considerar dentro del orden de magnitud del límite de detección del equipo, por lo que, independientemente de las morfologías superficiales diferentes, se puede decir que su composición es la misma.

\subsection{Análisis por DRX}

Con el fin de determinar las fases cristalinas presentes en las costras de corrosión se llevó a cabo un análisis por DRX. Dicho análisis se realizó con un fragmento de la muestra de costra 2, que se molió en mortero de ágata hasta un tamaño de grano inferior a $30 \mu \mathrm{m}$. Se identificaron óxidos Maghemita $\left(\mathrm{Fe}_{2} \mathrm{O}_{3}\right)$ e hidróxidos Goethita $(\mathrm{FeO}(\mathrm{OH}))$ de hierro (Fig. 9). Según la intensidad de sus reflexiones, la Maghemita presenta un mayor grado de cristalinidad que la Goethita. Es decir, en la costra de corrosión se ha formado mayor proporción de óxidos de hierro que de hidróxidos de hierro, puesto que los picos de difracción de los anteriores presentan mayor intensidad.

\section{DISCUSIÓN}

Los elementos metálicos observados in situ en la estación de ferrocarril de Aranjuez, así como las muestras estudiadas, indican que dichos materiales han sufrido un proceso de corrosión intenso. Este proceso ha dado lugar a una degradación química severa, en general, y extremadamente severa en un porcentaje relativamente elevado del conjunto de elementos (Tabla I).

La climatología local de Aranjuez (temperatura media $13,8^{\circ} \mathrm{C}$, promedio de 68 días de lluvia al año),

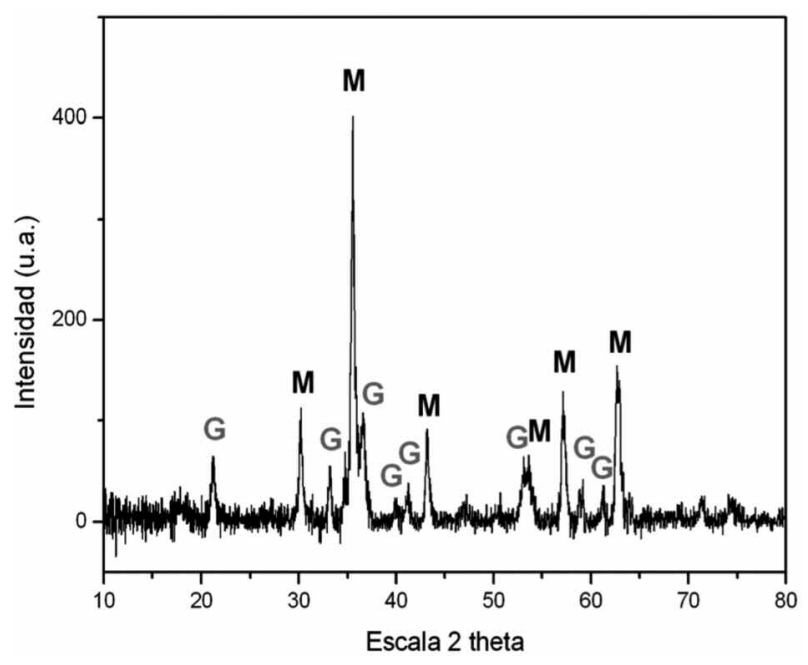

Figura 9. Difractograma de rayos $X$ de la muestra de costra 2. Fases cristalinas: M, Maghemita $\left(\mathrm{Fe}_{2} \mathrm{O}_{3}\right) ; \mathrm{G}$, Goethita $(\mathrm{FeO}(\mathrm{OH}))$.

Figure 9. X-ray diffraction pattern of sample crust 2. Crystalline phases: $M$, Maghemite $\left(\mathrm{Fe}_{2} \mathrm{O}_{3}\right)$; $\mathrm{G}$, Goethite $(\mathrm{FeO}(\mathrm{OH}))$.

determina porcentajes medios de humedad relativa muy elevados (del orden del 75 \%), en comparación con los valores medios del resto de la Comunidad de Madrid, como se puede esperar de una zona muy próxima al río Tajo y con altitud en la zona de la estación de ferrocarril de $494 \mathrm{~m}$ sobre el nivel del mar en Alicante. En una primera fase ${ }^{[6]}$, la interacción de este tipo de atmósfera ${ }^{[7}$ y 8$]$ con los elementos metálicos determina un ataque químico en medio hidrolítico $^{[9]}$ focalizado en la superficie de los materiales ${ }^{[10]}$. Sin embargo, la presencia de gruesas costras de corrosión desprendidas por completo de la correspondiente matriz metálica y la presencia de costras más delgadas adheridas a dichos metales sugiere que, además del proceso de ataque hidrolítico, se ha superpuesto otro mecanismo de alteración que ha intensificado la corrosión. El análisis metalográfico y por MEBEC/EDX de las muestras, así como las fases detectadas por DRX en las costras de corrosión, permiten deducir que el proceso de corrosión ha tenido lugar en un medio contaminado o de tipo industrial. En este caso al proceso de ataque hidrolítico se suma el ataque en medio ácido ${ }^{[11]}$, proporcionado por la combinación de la humedad ambiental con gases contaminantes de características ácidas (dióxido de azufre, óxidos de

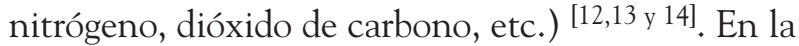
mayoría de los casos, esos gases contaminantes proceden de la combustión de motores que utilizan combustibles fósiles (posiblemente de locomotoras de carbón y de motor Diesel que funcionaron durante décadas). 
El mecanismo del ataque químico producido se puede esquematizar como sigue. La reacción que tiene lugar cuando el acero se expone al aire húmedo en condiciones neutras es la siguiente:

$$
\mathrm{Fe}^{0}(\mathrm{~s}) \rightarrow \mathrm{Fe}^{2+}+2 \mathrm{e}^{-} \mathrm{E}=-0,44 \mathrm{~V}
$$

Esta reacción de oxidación del hierro metálico $\left(\mathrm{Fe}^{0}\right)$ se produce cuando existe otra especie química que se reduce. En medio neutro son las moléculas de oxígeno $\left(\mathrm{O}_{2}\right)$ disueltas en las pequeñas gotas de agua $\left(\mathrm{H}_{2} \mathrm{O}\right)$ las responsables de dicha reducción ${ }^{[15]}$ :

$$
1 / 2 \mathrm{O}_{2}(\mathrm{~g})+\mathrm{H}_{2} \mathrm{O}+2 \mathrm{e}^{-} \rightarrow 2 \mathrm{OH}^{-} \mathrm{E}=+0,40 \mathrm{~V}
$$

La suma de las dos semirreacciones anteriores resulta:

$$
\mathrm{Fe}^{0}(\mathrm{~s})+1 / 2 \mathrm{O}_{2}(\mathrm{~g})+\mathrm{H}_{2} \mathrm{O} \rightarrow \mathrm{Fe}(\mathrm{OH})_{2}(\mathrm{~s})
$$

Esta reacción constituye la etapa incipiente del proceso de corrosión del acero. Sin embargo, el hidróxido de hierro (II) $\mathrm{Fe}(\mathrm{OH})_{2}$ expuesto al aire, se oxida fácilmente a hidróxido de hierro (III) $\mathrm{Fe}(\mathrm{OH})_{3}$, de modo que la reacción total para el proceso de corrosión es:

$$
2 \mathrm{Fe}^{0}(\mathrm{~s})+3 / 2 \mathrm{O}_{2}(\mathrm{~g})+3 \mathrm{H}_{2} \mathrm{O} \rightarrow 2 \mathrm{Fe}(\mathrm{OH})_{3}(\mathrm{~s})
$$

El producto final es de color pardo rojizo, no se adhiere a la matriz de acero y, por tanto, se desprende fácilmente formando las denominadas costras de corrosión o herrumbre ${ }^{[16 \text { y } 17]}$.

Cuando el medio es ácido, debido a la presencia simultánea de humedad y de contaminantes gaseosos de características ácidas $\left(\mathrm{SO}_{2}, \mathrm{NO}_{\mathrm{x}}, \mathrm{CO}_{2}\right.$, etc. $)$, son los iones $\mathrm{H}^{+}$procedentes de dicho medio los que se reducen a hidrógeno elemental $\left(\mathrm{H}^{0}\right)$ según la reacción:

$$
2 \mathrm{H}^{+}(\mathrm{I})+2 \mathrm{e}^{-} \rightarrow \mathrm{H}_{2}(\mathrm{~g}) \quad \mathrm{E}=0,00 \mathrm{~V}
$$

Esta semirreacción de reducción sumada con la semirreacción de oxidación del hierro metálico $\left(\mathrm{Fe}^{0}\right)$ resulta:

$$
\mathrm{Fe}^{0}(\mathrm{~s})+2 \mathrm{H}^{+} \rightarrow \mathrm{H}_{2}(\mathrm{~g})+\mathrm{Fe}^{2+}
$$

Los iones hierro (II) $\left(\mathrm{Fe}^{2+}\right)$, reaccionan posteriormente con el agua de la atmósfera, para formar los hidróxidos de Fe (II) y de Fe (III) $\left(\mathrm{Fe}(\mathrm{OH})_{2}\right.$ y $\mathrm{Fe}(\mathrm{OH})_{3}$, respectivamente).

El proceso global de degradación ${ }^{[18]}$ comienza en las picaduras superficiales de la pintura que avanza interconectándolas y desprendiendo parcialmente la capa de pintura. En una etapa posterior el avance de la corrosión continúa incluso por debajo de los restos de pintura ${ }^{[19]}$ y progresa rápidamente al abrigo del aire, dando lugar a una costra de corrosión esponjosa que termina de desprender la pintura y a la vez protege el frente de avance de la corrosión hacia el metal inalterado. Cuando el espesor de la costra y su peso superan las fuerzas de adherencia a la matriz metálica, la costra se desprende dejando una capa de costra relativamente delgada sobre el metal, que continúa y repite el ciclo de modo indefinido. Tal es el caso de las perforaciones que se han observado.

En el caso particular de los pilares y correas hay que tener en cuenta que la conducción de aguas se efectúa por un bajante ubicado en el interior de los pilares y que cualquier avería o deterioro de la tubería puede haber originado derrames de agua que anegarían la cubierta y el interior de los pilares. Esto podría explicar el lamentable estado de conservación de un porcentaje muy elevado de correas y la avanzada corrosión de determinados pilares y/o de determinadas caras de pilares.

Por otro lado, se ha observado un estado de conservación más deficiente en los elementos de los andenes $\mathrm{A}$ y $\mathrm{B}$, que son los más expuestos a las inclemencias meteorológicas, y los del extremo Alicante a la altura de los andenes D y E, que también se hallan muy expuestos por tratarse de una esquina. También se puede explicar que la parte superior de los pilares siempre presenta una corrosión más avanzada que las correspondientes partes inferiores. Asimismo, resulta lógico que las correas en peor estado de conservación sean aquellas más próximas a los pilares que muestran una corrosión avanzada, pues presumiblemente el agua derramada se distribuiría tanto por la cubierta como se deslizaría por el pilar hacia su base. Sin embargo, los pilares mejor conservados, desde el punto de vista de la corrosión de los metales, son aquellos en los que probablemente se suprimió la conducción de aguas para habilitar los pasos inferiores entre andenes. Dichos pilares son los que están apuntalados y presentan hundimiento.

\section{INTERVENCIÓN Y CONSERVACIÓN PREVENTIVA}

\subsection{Elementos metálicos}

Los resultados analíticos del presente trabajo indicaron la conveniencia de eliminar de los elementos metálicos, todas las partes afectadas severamente por cualquier proceso de corrosión (degradación manifiesta en forma de picaduras, perforaciones, roturas, 
abombamientos, faltas, etc. desde intensidad media hasta muy avanzada o completa, ver tabla I), en tanto podrían afectar de modo directo y grave tanto a la seguridad arquitectónica del conjunto de edificios, como a la del personal y los viajeros. Los resultados de los análisis efectuados sugieren que los materiales preferidos de sustitución sean los siguientes:

- Para elementos de pilares, correas, vigas, pletinas y otros en acero: acero común al carbono, con bajo contenido de carbono, de matriz ferrítico-perlítica, laminado en caliente.

- Para elementos de fundición: fundición gris de matriz perlítica.

- Para roblones: tornillos de acero de alta resistencia con cabeza redondeada similar a la parte más externa de los roblones originales.

Los pilares con un estado de conservación aceptable o con patologías de corrosión que responden positivamente a un proceso de restauración o sustitución parcial de materiales, deberían conservarse tanto como sea posible. En los pilares donde la corrosión ha afectado seriamente la integridad de todas o casi todas sus caras, es recomendable una sustitución de los elementos degradados. Es recomendable una sustitución de las correas que presentan un estado de conservación deficiente o muy deficiente. Esto supone la sustitución prácticamente global de las correas de todas las cubiertas.

Las piezas de fundición que se encuentran íntegras podrían conservarse una vez efectuada su limpieza, restauración y pintura. Las piezas que faltan deberían reponerse con piezas de fundición fabricadas a partir de un molde extraído de los modelos originales que se encuentran en mejor estado de conservación. Lo mismo puede decirse para las diversas piezas troqueladas que decoran los aleros.

\subsection{Pinturas}

En lo que respecta a los sistemas de protección superficial de elementos metálicos mediante pinturas (imprimaciones y sistema de pintura multicapa) es conveniente un tratamiento previo de acondicionamiento de las superficies y una posterior aplicación del sistema multicapa. El acondicionamiento debería incluir la eliminación física de todos los productos de la corrosión ${ }^{[20]}$. A este tratamiento previo debería seguir una limpieza cuidadosa por chorro de arena $\left(\mathrm{SiO}_{2}\right)$ o similar (profundidad no superior a $100 \mu \mathrm{m}$ ), que asegure tanto una superficie metálica virgen como un aumento de su superficie específica, lo que mejoraría la adherencia de las capas de imprimación y pintura. Tanto las capas de imprimación como las de pinturas deben estar exentas de cromatos $\left(\mathrm{CrO}_{4}{ }^{2}\right)$ o de cualquier compuesto que contenga $\mathrm{Cr}(\mathrm{VI})$, así como de óxidos de plomo (entre ellos minio $\mathrm{Pb}_{3} \mathrm{O}_{4}$ ) u otras especies tóxicas y/o contaminantes del medio ambiente ${ }^{[21]}$. Como alternativas se podrán utilizar imprimaciones basadas

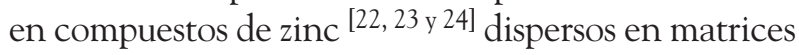
de tipo epoxi. El espesor adecuado podría estar comprendido entre 30 y $70 \mu \mathrm{m}$. La capa de imprimación se debería secar en $1 \mathrm{~h}$ aproximadamente.

La capa protectora intermedia tendría que ser específica para estructuras metálicas expuestas indefinidamente a la intemperie, por lo tanto de durabilidad prolongada y resistente a medios húmedos y/o contaminados. Su adherencia y compatibilidad con la capa de imprimación debe ser buena, presentando una flexibilidad adecuada respecto a la capa de pintura de acabado.

La capa de pintura de acabado, cuyo espesor recomendado oscila entre 50 y $100 \mu \mathrm{m}$, debería poseer una elevada resistencia frente a los agentes atmosféricos y una estabilidad probada en cuanto a color, brillo y textura. Sus características mecánicas deberán garantizar resistencia al rayado, abrasión, impactos y agresiones que induzcan al deslaminado. Su durabilidad química estaría garantizada frente a disolventes, grasas, productos de limpieza y, preferiblemente, a las pinturas comúnmente usadas en pintadas vandálicas.

\subsection{Conservación preventiva}

La conservación preventiva de todos los elementos metálicos debe estar sujeta a una revisión periódica y relativamente frecuente de la pintura protectora y de los primeros indicios de picaduras u otros signos de alteración superficial. Las inspecciones visuales deberían realizarse cada tres meses, especialmente durante la temporada húmeda y fría. La detección de patologías submicroscópicas, y a ser posible microscópicas, podría llevarse a cabo una vez al año, coincidiendo con una campaña de revisión detallada de la imprimación y del sistema multicapa de la pintura protectora. La restauración de elementos alterados o la sustitución de elementos degradados deben tener lugar tan pronto como sea posible, una vez realizado el diagnóstico correspondiente.

La mejor acción preventiva consiste en una monitorización y evaluación crítica de las condiciones ambientales particulares de la estación de ferrocarril. Para ello sería necesario la instalación de una unidad de monitorización atmosférica que incluya una estación meteorológica, que recogiera información sobre los siguientes parámetros: humedad relativa, 
temperatura, contenido de $\mathrm{O}_{2}, \mathrm{NO}_{\mathrm{x}}$ y $\mathrm{CO}_{2}$, partículas sólidas en suspensión, velocidad del viento y volumen de lluvias. La instalación de sensores ópticos de control de la acidez ambiental ${ }^{[25]}$ en dicha unidad de monitorización permitiría evaluar anticipadamente el riesgo de lluvia ácida y su incidencia en la corrosión de los elementos metálicos.

\section{CONCLUSIONES}

- La estación de ferrocarril de Aranjuez presenta un volumen elevado de elementos metálicos que se integran en la estructura arquitectónica y además conforman su estética histórica y ornamental. Por ello, el mantenimiento y conservación del carácter singular que aportan dichos elementos metálicos, se destaca como un requerimiento lógico y con la proyección futura de preservación de una característica singular del patrimonio ribereño.

- La práctica totalidad de los elementos metálicos han sufrido un proceso de corrosión intenso. Los análisis metalográficos y por MEBEC/EDX, así como las fases detectadas por DRX en las costras de corrosión, permiten deducir que dicha corrosión se corresponde con un ataque propiciado por ambientes de tipo industrial y/o contaminado y combinado con humedad relativa elevada. En este caso al proceso de ataque hidrolítico se suma el ataque en medio ácido, proporcionado por la combinación de la humedad ambiental con gases contaminantes de características ácidas $\left(\mathrm{SO}_{2}, \mathrm{NO}_{\mathrm{x}}, \mathrm{CO}_{2}\right.$, etc. $)$.

- Los criterios de intervención que se pueden adoptar de acuerdo con este trabajo son coherentes con el estado presente de deterioro y, frecuentemente, de degradación profunda en el que se encuentran los materiales objeto de estudio. Se considera adecuada y recomendable una sustitución de los materiales originales por los materiales recomendados cuando el grado de corrosión de aquellos supera la denominación "media", e incluso cuando, efectuado un ensayo de limpieza y restauración en los elementos con corrosión "media", se constate la ineficacia del procedimiento y/o su dudosa eficacia futura y preventiva.

\section{Agradecimientos}

Los autores del presente trabajo desean expresar su agradecimiento a INECO-TIFSA, autores del PROYECTO CONSTRUCTIVO DE REHABILITACIÓN DE LA ESTACIÓN DE FERROCARRIL DE ARANJUEZ
(MADRID), coordinador general A. Ranz Casares, autores técnicos responsables J. Cortezo García y J. Contreras Plaza, autores del informe histórico T. Prieto Palomo y P.J. Martín Blanco.

Los autores del IH-CCHS del CSIC agradecen la financiación parcial del Programa Consolider Ingenio 2010 ref. TCP CSD2007-00058, del Programa Geomateriales ref. S209/Mat-1629 y del proyecto MAT-CICYT ref. 2006-04486. Asimismo, agradecen el apoyo profesional de la Red Temática del CSIC de Patrimonio Histórico y Cultural.

\section{REFERENCIAS}

[1] Servicio Histórico Del COAM: M. Lasso de la Vega Zamora (coordinador), P. Rivas Quinzaños, A. Sanz Hernando, I. Esteban Maluenda y M. Fernández Ferreras. Arquitectura y Desarrollo Urbano: Comunidad de Madrid. Tomo IX. Zona Sur (Aranjuez). Ed. Dirección General de Arquitectura y Vivienda. Consejería de Medio Ambiente y Ordenación del Territorio, Fundación Caja de Madrid y Colegio Oficial de Arquitectos de Madrid. $1^{\underline{a}}$ ed. Madrid, España, 2004.

[2] T. Tortella Casares, Una guía de fuentes sobre las inversiones extranjeras en España (1780-1914), Ed. Banco de España, Madrid, España, 2000, p. 99.

[3] R.Peironcely, Presupuesto de gastos para el establecimiento de una vía apartadero y edificio para el servicio de viajeros en la vía general del triángulo. Madrid, España, 1904.

[4] T. Prieto Palomo y P. Martín Blanco, Informe Histórico de la Estación de Ferrocarril de Aranjuez. Proyecto constructivo de rehabilitación de la estación de ferrocarril de Aranjuez (Madrid). INECO-TIFSA. Coordinador general A. Ranz Casares, autores técnicos J. Cortezo García y J. Contreras Plaza. Madrid, España, 2008

[5] C. Flores, Hogar y Arquitectura 67 (1966).

[6] W. Han, G.C. Yu, Z.Y. Wang y J. Wang, Corr. Sci. 49 (2007) 2.920-2.935.

[7] H. Katayama, K. Noda, H. Masuda, M. Nagasawa, M. Itagaki y K. Watanabe, Corr. Sci. 47 (2005) 2.599-2.606.

[8] S. Hoerle, F. Mazaudier, P. Dillmann y G. Santarini, Corr. Sci. 46 (2004) 1.431-1.465.

[9] S. Feliu, M. Morcillo y S. Feliu Jr., Corr. Sci. 34 (1993) 403-414.

[10] I.S. Cole, W.D. Ganther, J.D. Sinclair, D. Lau y D. A. Paterson, J. Electrochem. Soc. 151 (2004) B627-B635. 
[11] C. Arroyave, F.A. López y M. Morcillo, Corr. Sci. 37 (1995) 1.751-1.761.

[12] D. De La Fuente, B. Chico, M. Morcillo y J. Simancas, AFINIDAD 62 (519) (2005) 479-486.

[13] C. Arroyave y M. Morcillo, Corr. Sci. 37 (1995) 293-305.

[14] M. Morcillo, L.S. Hernández, E. Otero y S. Feliu, J. Oil \& Colour Chemists Assoc. 72 (1989) 402-406.

[15] H. Nagano, T. Doi y M. Yamashita, Mater. Sci. Forum 289-2 (1998) 127-133.

[16] M. Kimura, T. Suzuki, G. Shigesato, H. Kihira y S. Suzuki, ISIJ Int. 42 (2002) 1.534-1.540.

[17] E. Otero, J.A. González, B. Chico y M. Morcillo, Mater. \& Corr. 53 (2002) 807-812.
[18] E. Almeida, M. Morcillo, B. Rosales y M. Marrocos, Mater. \& Corr. 51 (2000) 859-864.

[19] D. De La Fuente, J. Simancas y M. Morcillo, Prog. Org. Coat. 46 (2003). 241-249.

[20] D. De La Fuente, M. Bohm, C. Houyoux, M. Rohwerder y M. Morcillo, Prog. Org. Coat. 58 (2007) 23-32.

[21] E. Almeida, D. Santos, F. Fragata, O. Rincón y M. Morcillo, Mater. \& Corr. 52 (2001) 904-919.

[22] D. De La Fuente, B. Chico y M. Morcillo, Rev. Metal Madrid Suppl. S (2003) 129-136.

[23] S. Feliu, M. Morcillo y S. Feliu Jr., Corrosion 57 (2001) 591-597.

[24] S. Feliu, M. Morcillo, J.M. Bastidas y S. Feliú Jr., J. Coat. Technol. 65 (1993) 43-48.

[25] M.A. Villegas, Patente CENIM-CSIC P200602403 (2006). 